\title{
Gas Kinematics from Spectroscopy with a Wide Slit: Detecting Nuclear Black Holes
}

\author{
Witold Maciejewski and James Binney \\ Theoretical Physics, University of Oxford
}

\begin{abstract}
Motivated by STIS observations of more than 50 nearby galactic nuclei, we consider long-slit emission-line spectra when the slit is wider than the instrumental PSF, and the target has arbitrarily large velocity gradients. The finite width of the slit generates complex patterns in the spectra that can be misinterpreted as coming from various physically distinct nuclear components, but when interpreted correctly, they can have considerable diagnostic power. For a thin disk in circular motion around a central galactic black hole $(\mathrm{BH})$, a characteristic artifact occurs in the spectrum at the outer edge of the BH's sphere of influence. It betrays the presence of a $\mathrm{BH}$, and allows us to develop a new method for estimating its mass, which gives higher sensitivity to $\mathrm{BH}$ detection than traditional methods.
\end{abstract}

\section{Introduction}

Observations with long-slit spectrographs often use a slit that is wider than the FWHM of the instrumental point-spread-function (PSF). This practice is conventionally considered to enhance the signal-to-noise ratio $(\mathrm{S} / \mathrm{N})$ of the data at the price of what may be an insignificant loss in velocity resolution. However, when the target has steep velocity gradients, the use of a wide slit can have more subtle effects, because the position and velocity information become entangled along the dispersion direction. If these effects are not recognized in the data, a misleading impression of the structure of the target can be inferred. When the effects of a wide slit are recognized, however, they can increase the diagnostic power of the spectrum over that of a narrow-slit spectrum of equal S/N.

This study has been motivated by our participation in STIS observations of more than 50 nearby galactic nuclei (see Marconi et al. 2000, this volume), therefore we focus on the effect that a wide slit has on the emission-line spectra from gaseous disks around a central massive black hole (BH). However, the use of a wide slit affects spectra of any objects with steep velocity gradients (e.g. shocks or contact discontinuities).

\section{A slit wider than the PSF}

The conventional approach to the determination of the $\mathrm{BH}$ mass from emissionline spectra involves fitting a PSF-convolved circular-speed curve to the observations well into the nucleus of the galaxy, where the $\mathrm{BH}$-related velocity rise 

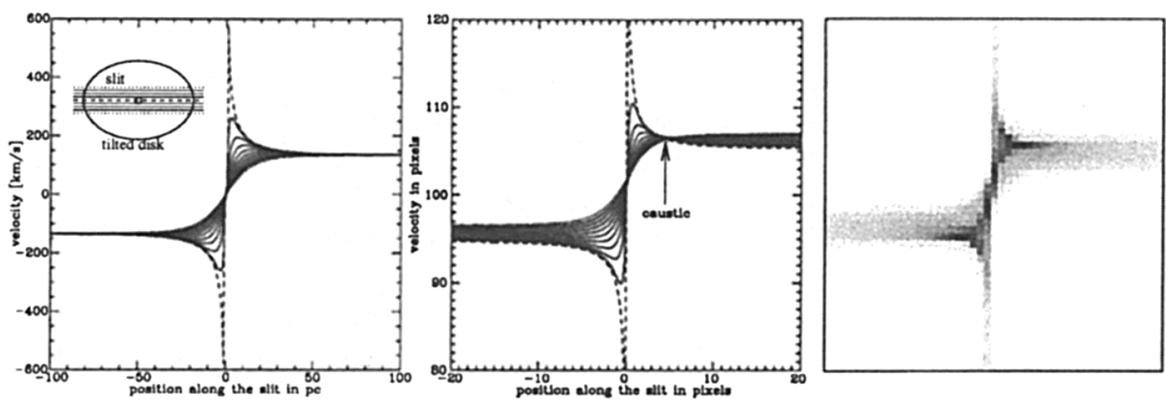

Figure 1. Left: Position-velocity diagram for the disk inclined at $60^{\circ}$, rotating in a potential of a $10^{8} \mathrm{M}_{\odot} \mathrm{BH}$ plus extended density distribution $\sim R^{-1.8}$. Velocities are sampled along cuts parallel to the line of nodes, shown in the inset at upper left. Center: The light pattern on the spectrograph's detector for G750M grating on STIS (only the top half of the slit is sampled). Right: The light from entire slit integrated within the detector's pixels.

inwards becomes apparent. A wide slit samples off-center velocities (solid lines in the left panel of Fig.1) in addition to the central rotation curve (the dashed line). When the disk is observed in some emission line through a long-slit spectrograph, the pattern of intensity resembles the left panel of Fig.1, but it is modified by the geometry of the spectrograph's optics: the diffraction pattern produced by light that enters near one edge of the slit (dotted line) is displaced with respect to light of the same frequency that enters at the corresponding point on the other edge of the slit. Thus the difference in position across the slit is seen on the detector as an instrumental velocity offset. To reflect the light pattern on the detector, the left panel of Fig.1 has to be modified by shifting each sample line in the dispersion direction by a constant amount, different for each line, because each line samples a given position across the slit.

The result for one half of the slit is presented in the central panel of Fig.1 (a plot for the other half is point-symmetric to this one). We see that in the presence of a velocity gradient across the slit, the instrumental velocity offset competes with the Doppler shifts: this offset wins at large radii, and at small radii the Doppler shifts take over. In between, both factors are of similar strength: light from all positions across the slit converges at one effective velocity, forming the caustic. At radii interior to the caustic, two maxima in the light distribution are present (Fig.1, right): one from the slit center (showing Keplerian rise), and one from the slit edges (passing through zero velocity at the nucleus). Thus the position-velocity diagrams for rotating disks are rich in structure; the information contained in them is lost in the conventional fitting of a Gaussian emission line profile, because this reduces them to a single position-velocity line. Below we explore the entire 2-dimensional light distribution.

\section{A new BH mass estimator}

The position of the caustic along the slit is quite independent of the stellar density profile, and it can indicate the presence, and betray the mass $M_{\bullet}$, of 
the $\mathrm{BH}$. A wide slit contains many narrow slits within it, and it can provide information that otherwise would need two offset thin slits. If the caustic occurs at a position $\alpha$ down the $2 \delta$-wide slit, then velocities at the slit center $\left(v_{c}\right)$ and at the slit edge $\left(v_{e}\right)$ are related by $D v_{c}(\alpha)=D v_{e}(\alpha)+B \delta$, where $B$ is the plate scale in the dispersion direction, and $D$ is the spectrograph's dispersion. If the only unknowns in the problem are the disk's inclination angle $i$ and $M_{\bullet}$, then this additional constraint allows us to recover $i$ and $M_{\bullet}$ independently, rather than $M_{\bullet} \sin i$. In the case of the slit placed along the line of nodes, the solution takes a particularly simple form:

$$
M_{\bullet}=\frac{\alpha d}{G}\left(\frac{y}{D \sin i}\right)^{2}, \quad \cos i=\frac{\delta}{\alpha}\left(\left(1-\frac{B \delta}{y}\right)^{-4 / 3}-1\right)^{-1 / 2},
$$

where $y$ is the position of the caustic in the dispersion direction, $d$ is the distance to the galaxy, and $G$ is the gravitational constant.

In addition to recovering $i$ and $M_{\text {. independently, this method gives higher }}$ sensitivity to $\mathrm{BH}$ detection than traditional methods based on the Keplerian rise in velocity occurring inside the BH's sphere of influence, because it exploits an artifact at the outer edge of this sphere. For the same reason though, it only yields the mass within the radius of the caustic, and it cannot determine whether the measured mass comes from the BH. On the other hand, the very existence of the caustic comes from the steep velocity rise towards the nucleus, which itself is characteristic of a massive $\mathrm{BH}$. Thus the main advantage of our technique is in detecting $\mathrm{BHs}$ in galaxies for which we cannot achieve the resolution required to follow the Keplerian rise in velocities inwards.

\section{Inclined slits}

Since nuclear disks frequently lie in a plane that differs markedly from that of galaxy's main disk, one usually does not know the line of nodes before nuclear spectra are taken. When the slit lies along the line of nodes (like in Fig.1), cuts offset by $\pm \delta$ below and above the nucleus map to the same line in the positionvelocity plot. The instrumental velocity offset shifts these lines up and down, and generates two lines: $y=D v+B \delta$ and $y=D v-B \delta$. For the disk with an opposite sense of rotation, the two cuts are mapped into two other lines: $y=D(-v)+B \delta=-(D v-B \delta)$, and $y=D(-v)-B \delta=-(D v+B \delta)$, which are just mirror symmetries of the previous two. If the slit is not placed along the line of nodes, then equidistant cuts passing below and above the nucleus sample two different velocity fields: $v(\alpha)=-v(-\alpha)$, and it matters which is moved up and which down. Thus the long-slit spectrum of a rotating disk taken with a wide slit depends qualitatively on the sense of rotation of the disk. For the same disk, one spectrum displays the caustic clearer that the other, therefore in our method the detectability of a $\mathrm{BH}$ with a given spectrograph setup depends on the sense of rotation of its accretion disk.

In Fig.1, where the slit is placed along the line of nodes, the outer envelope of the light distribution near the nucleus is formed by light coming through the slit center. This is not so for inclined slits (Fig.2, left panel): there the outer envelope comes from the sides of the slit - the dashed line associated with 

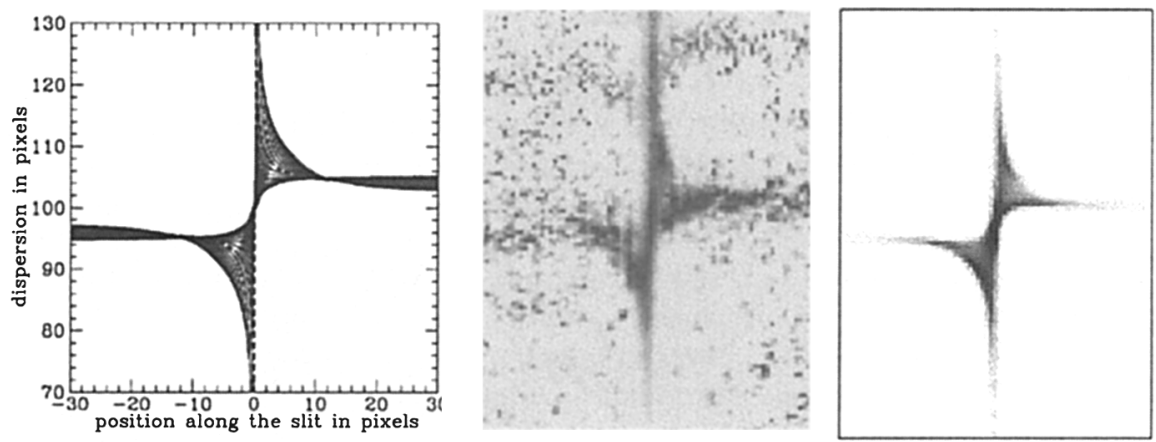

Figure 2. Left: Line sampling of our model of light distribution in the spectrum of a nuclear disk around a $\mathrm{BH}$ with parameters that we derived for M84 (the nuclear cut through the slit center is dashed). Middle: STIS $H \alpha$ emission-line spectrum of M84 (Bower et al. 1998). The dispersion direction is vertical. Right: light distribution in our model spectrum of M84 convolved with the PSF, and integrated over detector pixels (slit is offset by 0.03 arcsec from the nucleus).

the slit center has a much narrower peak. Consequently, fitting profiles from an infinitely thin slit to the observed light distribution would result in a considerable overestimation of the $\mathrm{BH}$ mass when the traditional method is being used.

\section{Confronting observations. Conclusions.}

The discussion above has been confined to the distribution of the principal maxima in the diffraction pattern produced on the spectrograph's detector. For our final models, we convolved these diagrams with the instrumental PSF on STIS approximated by a sum of Gaussians. Convolution was done by adding in intensity contributions from Airy disks formed on the slit and truncated by it.

We confronted then our predicted light pattern with the most detailed longslit spectrum of nuclear emission so far (M84, Bower et al. , 1998, ApJ, 492, L111): the last one shows two light maxima at radii close to the nucleus (Fig.2, middle panel). Although they were interpreted as coming from two physically distinct nuclear components, the observed light pattern has the same structure as our models in Fig.1. We interpret the point where the track of maximum light splits into two as the caustic, from which we estimate the disk to be inclined at $74^{\circ}$, and the $\mathrm{BH}$ mass to be $4 \times 10^{8} \mathrm{M}_{\odot}$, smaller than that of Bower et al. by a factor of 4 . This confirms our finding that $\mathrm{BH}$ masses derived by the traditional method may be overestimates. The left-right asymmetry of the observed spectrum comes from the slit not being centered on the nucleus: the right panel of Fig. 2 shows our predicted light pattern for the slit offset by .03 arcsec from the nucleus. Thus all features in the nuclear spectrum of M84 can be explained as coming from a thin disk in circular motion around the central $\mathrm{BH}$.

The finite width of the slit generates complex patterns in the spectra that can have considerable diagnostic power if they are modeled with adequate sophistication. They allowed us to develop a new method for estimating the $\mathrm{BH}$ mass that gives higher sensitivity to $\mathrm{BH}$ detection than traditional methods. 